\title{
Azimuth Phase Coding for Range Ambiguity Suppression in SAR
}

\author{
Dall, Jørgen; Kusk, Anders
}

Published in:

International Geoscience and Remote Sensing Symposium (IGARSS)

Link to article, DOI:

10.1109/IGARSS.2004.1370667

Publication date:

2004

Document Version

Publisher's PDF, also known as Version of record

Link back to DTU Orbit

Citation (APA):

Dall, J., \& Kusk, A. (2004). Azimuth Phase Coding for Range Ambiguity Suppression in SAR. In International Geoscience and Remote Sensing Symposium (IGARSS) (Vol. 3, pp. 1734-1737). IEEE.

https://doi.org/10.1109/IGARSS.2004.1370667

\section{General rights}

Copyright and moral rights for the publications made accessible in the public portal are retained by the authors and/or other copyright owners and it is a condition of accessing publications that users recognise and abide by the legal requirements associated with these rights.

- Users may download and print one copy of any publication from the public portal for the purpose of private study or research.

- You may not further distribute the material or use it for any profit-making activity or commercial gain

- You may freely distribute the URL identifying the publication in the public portal

If you believe that this document breaches copyright please contact us providing details, and we will remove access to the work immediately and investigate your claim. 


\title{
Azimuth Phase Coding for Range Ambiguity Suppression in SAR
}

\author{
Jørgen Dall and Anders Kusk \\ Electromagnetic Systems, Ørsted $•$ DTU, \\ Technical University of Denmark (DTU) \\ Kongens Lyngby, Denmark \\ jd@oersted.dtu.dk, ak@oersted.dtu.dk
}

\begin{abstract}
A novel ambiguity suppression technique is proposed. Range ambiguities in synthetic aperture radar (SAR) images are eliminated with an azimuth filter after having applied an azimuth phase modulation to the transmitted pulses and a corresponding demodulation to the received pulses. The technique excels by actually eliminating the ambiguities rather than just defocusing them as most other techniques do. This makes the proposed technique applicable to distributed targets. The range ambiguity suppression permits the pulse repetition frequency (PRF) to exceed the upper limit otherwise defined by the antenna elevation dimension. The fundamental antenna area constraint still applies, but the PRF can be chosen with more freedom. In addition to ambiguity suppression, potential applications include nadir return elimination and signal-to-noise ratio improvement.
\end{abstract}

Keywords-component; SAR; ambiguity; pulse repetition frequency; modulation; filter; nadir return; signal-to-noise ratio.

\section{INTRODUCTION}

Range ambiguities appear in SAR images if more than one transmitted pulse contributes to the backscattered signal that is received at any point in time [1]. The round-trip propagation time of the ambiguous signals differ from that of the desired signal by a (positive or negative) multiple of the reciprocal pulse repetition frequency (PRF). Therefore, the range ambiguities are images of strips parallel to the strip of interest, mutually separated by the slant range $R_{\mathrm{PRF}}$ that corresponds to the reciprocal PRF. These strips are weighted by the two-way antenna pattern and superimposed the image of the strip of interest.

The principal suppression of range ambiguities is accomplished by the antenna elevation pattern. The range attenuation contributes to the suppression of the range ambiguities coming from strips farther away than the strip of interest, whereas any other range ambiguity is enhanced by the range attenuation.

Range ambiguities appear if the antenna directivity in the direction of one or more of the ambiguous strips is not sufficiently low. Hence a narrow elevation beam width favors range ambiguity suppression. A given mapping geometry and $R_{\mathrm{PRF}}$ define a maximum acceptable elevation beam width and a minimum antenna width $W_{\min }\left(f_{\mathrm{PRF}}\right)[2]$.
On the other hand, adequate sampling of the Doppler signal implies a maximum pulse spacing equal to half the antenna length. Consequently, a given platform speed and PRF define a minimum antenna length $L_{\min }\left(f_{\mathrm{PRF}}\right)$. A shorter antenna makes the Doppler bandwidth exceed the PRF, thereby causing aliasing and azimuth ambiguities [2].

In combination, the minimum antenna width and the minimum antenna length define the minimum antenna area $A_{\min }$ $=W_{\min } L_{\min }$, which is independent of the PRF [2]. A SAR with a smaller antenna area suffers from either range ambiguities or azimuth ambiguities or both. A SAR with an antenna area equal to $A_{\min }$ does not have any ambiguities if the PRF matches the aspect ratio of the antenna. Otherwise, it suffers from range ambiguities or azimuth ambiguities, depending on whether the PRF is too low or too high. The same applies to a SAR with an antenna larger than $A_{\min }$, except that ambiguity-free imaging is accomplished with a whole range of PRFs.

SAR systems applying the displaced phase center (DPC) technique [3] are also subject to the antenna area constraint. A large swath width without range ambiguities is obtained with a low PRF, which in turn is permitted by a long antenna composed of multiple sub-antennas with separate receivers. Splitting the antenna into sub-antennas ensures a better azimuth resolution, defined by the sub-antenna length rather than the total antenna length.

The SCANSAR technique [2], on the other hand, circumvents the constraint imposed by the antenna area. A large swath width is achieved at the expense of a reduced azimuth resolution and a scanning capability.

Several ambiguity suppression techniques have been proposed. The finite depth of focus automatically defocuses the range ambiguities, thereby reducing their peak levels [4], [5]. However, their energy is not reduced, and so a poor integrated side lobe ratio (ISLR) results when distributed targets are mapped.

Modulation of successively transmitted pulses by orthogonal codes (e.g. up- and down chirps) spreads the ambiguities in the range direction. The technique significantly reduces the ambiguity peaks and performs well when bright point-like targets are mapped. However, the ambiguity energy is again unchanged and the technique is not suitable for distributed targets [1], [6]. 
Another range ambiguity suppression technique based on modulation / demodulation in azimuth has previously been proposed [5]. Using Parseval's theorem, it can easily be shown that with this technique the point target response of the range ambiguities is changed such that the main lobe is suppressed but the sidelobe level is increased and the total energy is unchanged.

A range ambiguity problem can easily be converted into an azimuth ambiguity problem simply by reducing the PRF. Therefore, availability of an efficient azimuth ambiguity suppression technique would to some extent solve the range ambiguity problem as well.

Azimuth ambiguities can be suppressed with a technique based on the concept of ideal filters [7]. This technique is well suited to point targets, but azimuth ambiguity fading causes problems with distributed targets. The same applies to the recursive technique suggested by Massonnet and Adragna [8]. Another class of azimuth ambiguity suppression techniques is based on "Selective Filtering" [9], [10]. The processed Doppler bandwidth is reduced in order to exclude the strongest parts of the ambiguity spectra. However, a coarser azimuth resolution or a reduced number of looks results from the bandwidth reduction.

In this paper a novel range ambiguity suppression technique is proposed. The technique does not fundamentally circumvent the constraint imposed by the antenna area, but it reduces the minimum required antenna area somewhat. Most importantly, the technique permits PRFs exceeding the maximum frequency otherwise defined by the antenna width.

In Section II the principles of the proposed range ambiguity suppression technique are introduced. Section III demonstrates that an azimuth phase modulation with the required properties does exist, and the modulation is derived mathematically and analyzed. Finally, Section IV outlines different applications of the proposed technique.

\section{AMBIGUITY SUPPRESSION PRINCIPLE}

The proposed technique includes three steps:

- azimuth phase modulation of the transmitted pulses

- azimuth demodulation of the received pulses

- azimuth filtering

The first step impacts the SAR system, and therefore the technique can generally not be applied to data already acquired. The modulation multiplies every transmit pulse by a phase factor $\mathrm{e}^{j \varphi(n)}$, which is a function of the pulse number $n$. For satellite SAR systems, the round trip time greatly exceeds the pulse repetition interval, so pulse $n$ is backscattered from the strip of interest and received as pulse number $n+m$.

The demodulation completely cancels the modulation of the pulses backscattered from the strip of interest. This is accomplished with a phase factor of $\mathrm{e}^{-j \varphi(n-m)}$. Compared to the strip of interest, the $k^{\prime}$ th range ambiguity has an additional twoway propagation time equal to $k$ times the pulse repetition interval, and therefore the modulation / demodulation leaves a residual modulation phase
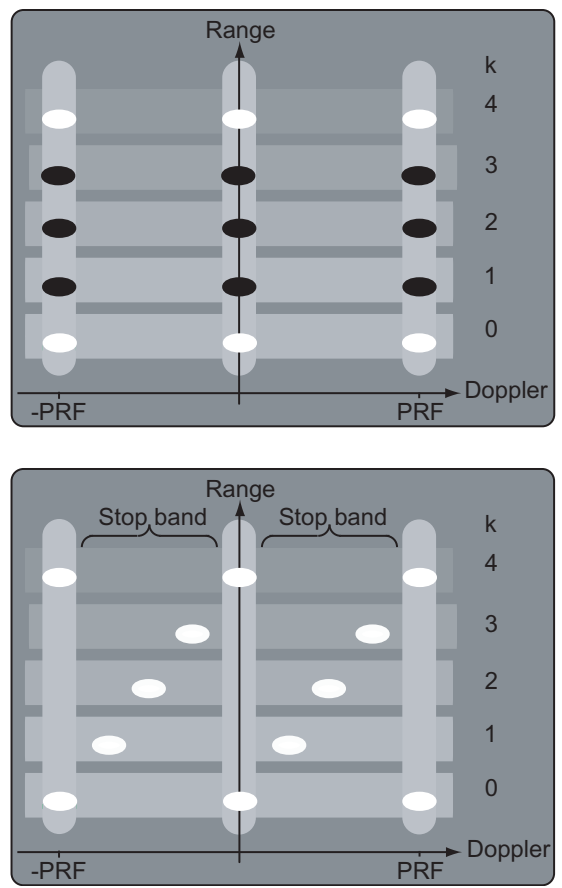

(b)

Figure 1. Doppler spectra: (a) initial, (b) after modulation and demodulation. Black spectra are not acceptable.

$$
\varphi_{\text {res }}(n)=\varphi(n-m-k)-\varphi(n-m)
$$

For the strip of interest $k=0$ and for potential ambiguities located between nadir and the strip of interest $k$ is negative.

Figure 1 illustrates the basic idea of the ambiguity suppression technique in the range-Doppler domain. The ambiguities are assumed harmless for $k>3$ and for simplicity a zero-Doppler geometry has been assumed. In Figure $1 \mathrm{~b}$ the residual modulation has shifted the three harmful ambiguity spectra such that they do not overlap the desired spectrum. This enables an azimuth filter to eliminate the ambiguities.

\section{MODUlation}

\section{A. Azimuth phase code}

In order to shift the spectra of the ambiguities, the residual phase must be a linear function of $n$. For instance, the first ambiguity is shifted by $f_{1}=f_{\mathrm{PRF}} / M$ if

$$
\varphi_{\text {res }}(n)=\varphi(n-m-1)-\varphi(n-m)=\frac{2 \pi n}{M}+\varphi_{c}
$$

Here $\varphi_{c}$ is an arbitrary constant. This equation can actually be solved, and the complete solution can be found by expansion and insertion of Eq. 2

$$
\begin{aligned}
\varphi(n) & =\varphi(0)+\sum_{i=1}^{n} \varphi(i)-\varphi(i-1) \\
& =\varphi(0)-\sum_{i=1+m}^{n+m}\left(\frac{2 \pi}{M} i+\varphi_{c}\right) \Leftrightarrow
\end{aligned}
$$




$$
\varphi(n)=\varphi(0)-n \varphi_{c}-\frac{2 \pi}{M} \sum_{i=1+m}^{n+m} i
$$

The last sum is an arithmetic series, the sum of which is $n(n+2 m+1) / 2$. Hence the modulation phase becomes

$$
\varphi(n)=\varphi(0)-n \varphi_{c}-\frac{\pi}{M} n(n+2 m+1)
$$

The simplest modulation is obtained by choosing the constants $\varphi(0)$ and $\varphi_{\mathrm{c}}$ such that

$$
\varphi(n)=-\frac{\pi}{M} n^{2}
$$

For arbitrary ambiguity number $k$, the residual modulation is found by inserting Eq. 4 in Eq. 1

$$
\varphi_{\text {res }}(n)=\frac{2 \pi}{M} k n-\frac{\pi}{M}\left(k^{2}+2 m k\right)
$$

The last term is a constant without importance, but the first term is linear in $n$ and shifts the Doppler spectrum of the $k^{\prime}$ th ambiguity by $k f_{\mathrm{PRF}} / M$. This means that the spectral shift is proportional to the ambiguity number.

Unfortunately, the $k$-dependence of the spectral shift is inevitable. If the same frequency shift could be applied to all ambiguities they would require smaller gaps in the desired spectrum. This in turn would require less oversampling and consequently a smaller $L_{\min }$ and $A_{\min }$.

As shown in the next two subsections the modulations defined by integer $M$ are particularly interesting. In this case, ambiguity spectra 1 to $M-1$ are equidistantly distributed in the gaps of the desired spectrum. This is illustrated in Figure 1c for $M=4$. Due to the periodic nature of the spectra, ambiguity number $k+I M$ is shifted by $(k+I M) f_{\mathrm{PRF}} / M$ modulo $f_{\mathrm{PRF}}=$ $k f_{\mathrm{PRF}} / M$. Hence, ambiguity number $k$ and $k+I M$ have the same shift for integer $I$.

For $M=2$, the proposed technique simplifies to the method that Giuli and Facheris [11] have proposed for polarimetric SAR systems alternately transmitting two orthogonal polarizations. Such systems are particularly sensitive to odd numbered co-polar range ambiguities contaminating a weak cross-polar signal.

\section{B. Permutation of the ambiguity spectra}

In practice, the strongest ambiguity spectrum should not be shifted to a position next to the desired spectrum. The ambiguity spectra adjacent to the desired spectrum are not completely eliminated if they overlap the desired spectrum or if they are in the transition band of the azimuth filter used to eliminate the ambiguities. Therefore the shifted ambiguity spectra may advantageously be permutated. For integer $M$, this can be accomplished by multiplying the modulation phase by a non-zero integer $J$

$$
\varphi(n)=-\frac{\pi J}{M} n^{2}
$$

Thereby the spectral shift of ambiguity number $k$ is increased by a factor of $J$

$$
f_{k}=\frac{J k f_{P R F}}{M} \bmod f_{P R F}
$$

Reducing $f_{k}$ modulo $f_{P R F}$ is equivalent to reducing $J k$ modulo $M$. Introducing $J$ in Eq. 6 results in a permutation of the ambiguity spectra if and only if $J$ and $M$ are mutually prime [12] (no common prime factor). Otherwise, one or more ambiguities are not shifted and cannot be eliminated.

The $\phi(M)$ elements in $S=\{1 \ldots M-1\}$ having no prime factor in common with $M$ constitute a subset denoted $S_{\mathrm{p}} . \phi(M)$ is known as Euler's totient function [12]. The $\phi(M)$ permutations that are generated with $J \in S_{\mathrm{p}}$ are all distinct (for instance, the shift of the first ambiguity, $J f_{\mathrm{PRF}} / M$, differs), and $J \notin S_{\mathrm{p}}$ does not contribute additional permutations because $(J+M) k$ modulo $M$ equals $J k$ modulo $M$. Consequently, $\phi(M)$ distinct permutations can be implemented with Eq. 7, but since $\phi(M) \leq M-1$ [12], this is but a subset of the $(M-1)$ ! permutations that exist.

\section{Code properties}

The modulation is computed modulo $2 \pi$ and it is periodic if

$$
\varphi(n+N L)=\varphi(n) \quad \bmod 2 \pi
$$

for all integers $n$ and $N$. If so, the period length is the smallest integer $L$ satisfying Eq. 8 . For $J \in S_{\mathrm{p}}$, and integer $M$, Eq. 8 is indeed satisfied, and the period is

$$
L= \begin{cases}M, & \text { Meven } \\ 2 M, & \text { Modd }\end{cases}
$$

This is seen by expanding Eq. 8 using Eq. 6

$$
\begin{aligned}
-\frac{\pi J}{M}(n+N L)^{2} & =-\frac{\pi J}{M} n^{2} & & \bmod 2 \pi
\end{aligned} \Leftrightarrow
$$

which, for $L$ given by Eq. 9, is satisfied for all integer $n$ and $N$.

For integer $M$ and $J$, the period length is small because, in practice, $M$ is small. For non-integer $M$ or $J$, the modulation phase is generally not periodic at all. Depending on the architecture of the SAR signal generator, a short period length may be crucial.

\section{APPLiCATIONS}

Referring to Figure 1a, a natural question is: why not just reduce the PRF by a factor of four? This would eliminate the three harmful range ambiguities and would not cause spectral overlap since the gaps in the desired Doppler spectrum are at least three times the Doppler bandwidth $B_{\mathrm{D}}$. Thus, range and azimuth ambiguities can be avoided by a proper choice of PRF because the antenna area $A$ is assumed to exceed $A_{\min }$. Actually, in most cases a reduced PRF is preferable to the proposed ambiguity suppression technique because the former results in a lower data rate, and the latter does not really circumvent the antenna area constraint, anyway. However, the following examples demonstrate the utility of the proposed technique. 


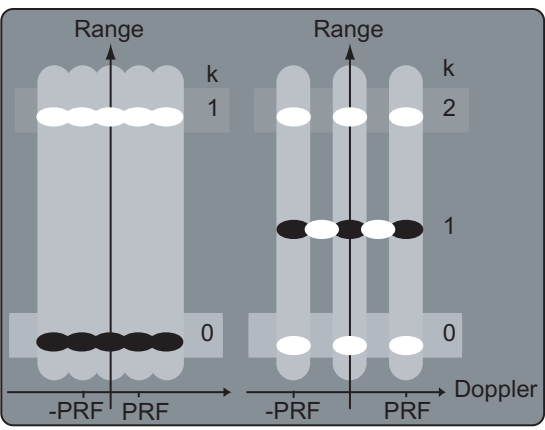

Figure 2. Suppression of azimuth ambiguity

In practice, $A_{\min }$ can be reduced somewhat. In Figure 2, $A$ is smaller than $A_{\min }$, so if the azimuth ambiguities are eliminated by increasing the PRF, a range ambiguity results. Neither the azimuth ambiguities nor the range ambiguity are acceptable. However, by applying the proposed ambiguity suppression technique after having doubled the PRF, the range ambiguity spectrum is shifted such that it is attenuated by both the antenna elevation pattern and the partial overlap with the desired spectrum, thereby suppressing it to an acceptable level.

Avoiding overlapping transmit and receive windows, nadir returns, and ambiguities severely constrains the possible PRFs. The proposed technique offers more flexibility in choosing a proper PRF. For instance, in Figure 3, where a squinted geometry is assumed, the range ambiguities are negligible except for number $k=-2$, which is particularly strong because of a nadir return. Rather than tuning the PRF such that this ambiguity is avoided, it can be shifted in frequency and eliminated. By using the permutation defined by $M=3$ and $J=$ 2 , the strong ambiguity is shifted by $-f_{\mathrm{PRF}} / 3$, thereby placing it in the stopband of the azimuth filter that is tailored to the desired spectrum.

Finally, range ambiguity suppression can be used to improve the signal-to-noise ratio (SNR) through an increased PRF. If, alternatively, the SNR is improved by increasing the pulse length, the processed swath width is decreased because the pulse compression results in more invalid samples. A PRF increase does not by itself imply an SNR improvement, but if the noise in the gaps of the Doppler spectrum is eliminated with an azimuth filter, an SNR improvement equal to the oversampling factor $f_{\mathrm{PRF}} / B_{\mathrm{D}}$ is achieved. This noise filtering is

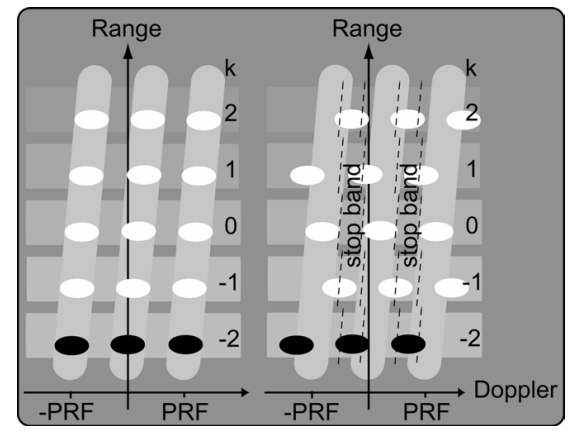

Figure 3. Elimination of nadir return at $k=-2$. automatically accomplished when the shifted range ambiguity spectra are eliminated. However, it is not always possible to increase the PRF. For a fixed pulse length, the duty cycle increases proportionally with the PRF, and most TWTs are confined to duty cycles on the order of $5 \%$. However, high power amplifiers based on solid state technology or microwave power modules (MPMs) do offer high duty cycles.

\section{CONCLUSIONS}

Unlike most other range ambiguity suppression techniques, the technique proposed in this paper eliminates the range ambiguities rather than defocus them, and hence it is well suited for distributed targets. The suppression of range ambiguities allows of higher PRFs than otherwise applicable with a given antenna width. The minimum antenna area, required for ambiguity-free operation, is reduced somewhat, but basically the area requirement is not circumvented.

Especially for space-borne SAR systems, many different constraints are imposed on the PRF, but the elimination of range ambiguities leaves more freedom in choosing the PRF. This freedom can, for instance, be used to increase the signalto-noise ratio of SAR systems characterized by a limited peak power but a high duty cycle.

The proposed technique impacts the SAR system in two different ways. First, the signal generator must be able to apply an azimuth phase modulation to the transmitted pulses. Typically, the phase modulation is periodic with a short repeat cycle. Secondly, azimuth oversampling is required and hence an increased data rate must be handled.

\section{REFERENCES}

[1] R.O. Harger, "Synthetic Aperture Radar Systems: Theory and Design", Academic Press, New York, 1970.

[2] F.T. Ulaby, R.K. More, and A.K. Fung, "Micrwave Remote Sensing", Vol. II, pp 652-660, Addison-Wesley Publishing Company, 1982.

[3] A. Currie and M.A. Brown, "Wide-swath SAR", IEE Proceedings Radar Sonar and Navigation Vol. 139, No. 2, pp. 122-135, 1992.

[4] K. Tomiyasu, "Tutorial review of synthetic-aperture radar (SAR) with applications to imaging of the ocean surface", Proc. of the IEEE, Vol. 66 No. 5, pp. 563-583, 1978.

[5] G. Krämer, "Suppression of Ambiguitites by Phase Coding", Proceedings of EUSAR, pp. 97-100, Cologne, May 2002.

[6] J. Márquez-Martinez and J. Mittermayer, "Analysis of Range Ambiguity Suppression methods in SAR by using a Novel Range Ambiguity Raw Data Simulator", Proc. of EUSAR, pp. 4077-4079, Cologne, May 2002.

[7] A. Moreira, "Suppressing the Azimuth Ambiguities in Synthetic Aperture Radar Images", IEEE Transactions on Geoscience and Remote Sensing, Vol. 31, No. 4, 1993.

[8] D. Massonnet and F. Adragna, "Synthetic Aperture radar: New Processing Concepts", Proceedings of IGARSS, pp. 1323-1326, Washington, May 1990.

[9] J.G. Mehlis, "Synthetic aperture radar range-azimuth ambiguity design and constraints", Proc. of IEEE International Radar Conference, pp. 143$152,1980$.

[10] A. Monti Guarnieri, "Space-Adaptive Ambiguity Suprression in SAR Images", Proc. of EUSAR, pp. 187-190, Ulm, May 2004.

[11] D. Giuli and L. Facheris, "Performance of interpulse Signal Coding in Interleaved-pulse Polarimetric SAR", European Transactions on Telecommunications and Related Technologies, Vol. 4, No. 5, 1993.

[12] H.J. Nussbaumer, "Fast Fourier Transform and Convolution Algorithms", pp. 6-12, Springer-Verlag, 1981. 